$\xi=-1$

\title{
Design and implementation of labview based bore well child rescue robot
}

\author{
M.Sujatha ${ }^{1 *}$, N.Prabakaran ${ }^{2}$, Selvakumar.R ${ }^{3}$ \\ ${ }^{1,2,3}$ Department of Electronics and Communication Engineering, KLEF, Vijayawada, Andhrapradesh \\ *Corresponding author Email: sujakarthik77@kluniversity.in
}

\begin{abstract}
An innovative method is proposed for rescuing a child from a borehole and to keep the child in conscious state. Children accidentally fall into the borehole which yielded water and subsequently left uncovered. A suitably strong cap of bright color to cover the mouth of the borehole will avoid such accidents. It is difficult and also risky process to rescue the trapped child after fallen into the borehole. In this paper, a robot is designed in such a way that it handles the trapped child carefully and monitors the child continuously. The robot structure consists of power supply, gear motors, oxygen concentrator, camera and micro controller, robotic arms, temperature sensor, audio player which has parents voice, heart beat monitoring system, lifting rods, servo motors. The condition of the child is captured with CCTV camera and monitored on a TV.

The proposed system is implemented using NI LabVIEW software. This robot type machine can rescue trapped body from the borehole in a highly secure way.
\end{abstract}

Keywords: Microcontroller Based Robot; Altitude Sensor; Virtual Instrumentation; Zigbee Wireless Technology.

\section{Introduction}

In Recent, the news of kids falling in abandoned bore well which are recklessly left revealed is often seen in various parts of India. These drag wells are the ones which are not of any utilization to the people as they do not deliver any water and in this manner individuals don't care to top them off or close them appropriately. Little children playing close such bore wells frequently fall into these bore wells and get caught.

Figure 1.1 shows the army members working manually for the bore well rescue. Protect of youngsters caught inside the bore wells is troublesome as well as a dangerous task. Rescue groups put in hours and now and again days in futile attempts to spare these little children. A ton of cash is also spent in these missions. As a rule they can't to save the children. Such occasions have happened numerous times before, and each time either the government or the foundation is faulted. The protect procedure to save the tyke from bore-well is a long and complicated process.

A little postponement in the protect can cost the victim his or her life. The safeguard group tries to approach the casualty from a parallel well that take around 20-60 hours to burrow. This confounded procedure makes $70 \%$ of the save operations fall flat. Not very many of the casualties have been spared in such mishaps. As of late some autonomous robots made advances on screen to take out the caught body efficiently. Be that as it may, the question rises, why these bots are not in real life in this present reality. This brings out security issue that how securely these robots handle the casualty. The safeguard operation for the most part comprises of three processes: Approaching the Casualty, Dealing with the body, Taking child out of the well. A customary self-ruling robot can easily play out the first and third operations in less time. But there is an awesome possibility for harm of casualty as they try attaching body organs and fabrics. To overcome these obstacles, we have outlined a drag well recue robot with propelled gear and gadgets. Along these lines the objective of this venture is to develop and plan a bore well protect framework which saves a trapped baby from bore well as well as manages outrageous safe handling of the casualty.

The plan of taking care of framework is made such that the casualty gets hurt minimally. This venture is a human controlled framework which with the help of remote camera gives a knowledge perspective of the victim and steps taken to accomplish this. The system consists of an apparatus get together to settle the framework with the inner dividers of the well, two simulated arms to hold the victim by his hands to keep from further sliding down inside the bore well, an enclosure like structure made up of thin strips is made to go through the dividers of the bore well to reach beneath the casualty and a security expand attached to the lower end of the pen to give the casualty support from underneath. The framework is remotely worked using wireless ZigBee innovation and utilizing remote camera we can see both sound and video on the PC.

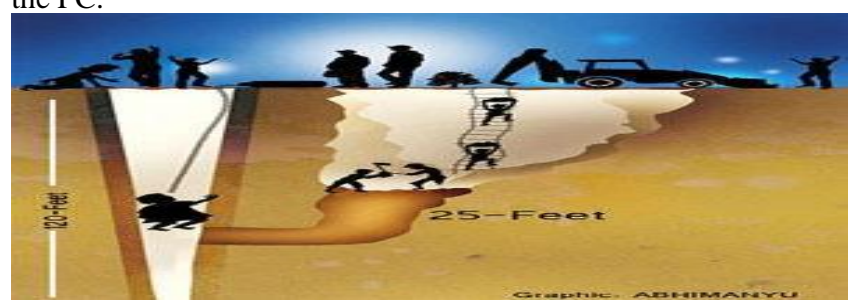

Figure 1.1 Army members working for the borehole rescue 


\section{Related Works}

The field of hunt and protect mechanical technology, while developing quickly in this decade, is still moderately exceptionally destructive have been proved. Outside of controlled conditions, people have just performed advanced control assignments when worked for safeguard operation .In typical save operation, a parallel pit is burrowed profound to accomplish the tyke and nearby openings are made to the dividers of bore well. A typical strategy used to discover the profundity of kid is the utilization of rope. One specific part of the save mechanical autonomy area facilitates the productive mix of exceedingly difficult fundamental research and application arranged advancements for expansive markets. This is the way that saves robots emphatically advantage from independence while there is a human on the up and up. The option answer for this issue is the utilization of mechanical frameworks which can move down the pipe and bring the subjected body out of it appropriately and securely.

Palwinder kaur, Ravinder kaur, Gurpeet singh [1] have proposed robots which have been exceptionally effective at control in reproduction and controlled conditions. It will be a light weight machine that will go down into the drag well pipe and hold the caught body methodically. In this option situation, there will be no prerequisite of burrowing any gap parallel to the drag well. The remotely controlled robot will go down the drag well and play out the activity. B.Bharathi, B.Suchitha Samuel [2] has proposed a framework comprise of a light weight machine that will go down into the drag well pipe and hold the caught body deliberately. A considerable measure of different bothers will likewise be dodged by this option strategy. The remotely controlled robot will go down the drag well and play out the activity of catching child outside the drag well utilizing 3 finger instrument. The 3 finger instrument is controlled utilizing portable PC. We are likewise utilizing temperature sensor to gauge the temperature of the infant caught encompassing.

K.Saran, S.Vignesh, Marlon Jones Loius [3] The essential utilization of robots incorporates looking for survivors, where uncommon perspectives can be seen with better human-robot connection. Normal technique took after by the safeguard group is first to discover the profundity of the youngster in the drag well by utilizing rope. In the wake of finding the profundity, a parallel pit is burrowed utilizing Earth moving vehicles. This strategy for safeguarding has taking after troubles, it takes up to 30 hours to burrow the parallel pit, at that point the kid would have passed on. Absence of oxygen inside the borehole and absence of perception causes the significant trouble amid the save operation. There is no such extraordinary hardware for saving the kid caught inside the borehole.

Manish Raj, P.Chakraborthy and G.C.Nandi [4] describes as the diameter of the bore well is narrow for any adult person and light goes ark inside it, the rescue task those situations is challenging. The robotic system designed will attach a harness to the child using pneumatic arms for picking up. A teleconferencing system will also attach to the robot for communicating with the child.

V.Venmathi, E.Poorniya, S.Sumathi [5] have proposed a safety balloon is introduced in order to provide extra safety. Once the lifting rod reaches a safe position under the child, an air compressor is operated to pump air to the bladder attached to the end of the lifting rod through an air tube that runs downwards inside the lifting rod.

Sridhar Palaniswamy [6] Pulling of child is made possible by special graspers, which can grasp the shoulder or the wrist or the ankle of the child. These have been specially designed and fabricated to provide open and close control at one end and facility to extend it by adding additional pipes

John Jose pottery [7] describes the facility to monitor the trapped child, supply oxygen and provide a supporting platform to lift up the child. The 1st motor placed at top turns a gear mechanism which, in turn, pushes 3 blocks arranged at 120 degrees from each other towards the side of the bore well. The 2nd motor placed below the plate turns the bottom shaft by 360 degrees, the helping to locate the gap through which the lifting rod passes. This is done with the help of a wireless camera attached to the lifting rod.

In all the above existing system, the presence of the child in the bore well is not identified by the rescue workers. It is more harmful during the rescue operation and also time consuming process and the oxygen present in the bore well is very low. This may lead to death of the child.

Forty five passing of youngsters have been accounted for in the nation since September 2009, from that we have just nineteen with the confirmation of a daily paper. In the time of 2012 Six year old kid was protected from the drag well, however later kicked the bucket in the healing centre because of wounds amid the safeguard operation and absence of therapeutic guide. Their passing are brought about because of revealed dry bore wells.

\section{Proposed Method}

The robot is operated through PC using wireless Zigbee technology and using wireless camera you can view both audio and video on the TV. This robot has a high power LED which acts as a light source when light intensity inside the pipe is low. Figure 3.1 shows the transmitter module and Figure 3.2 shows the block diagram of the robotic module. It is a low cost robot used to monitor the changes of different parameters in the industrial pipes.

\section{Robot to rescue of a child in a borehole 1. Transmitter}

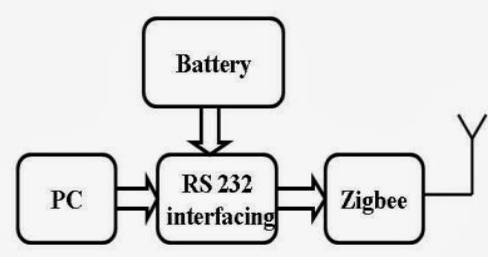

\subsection{Block diagram of Transmitter}

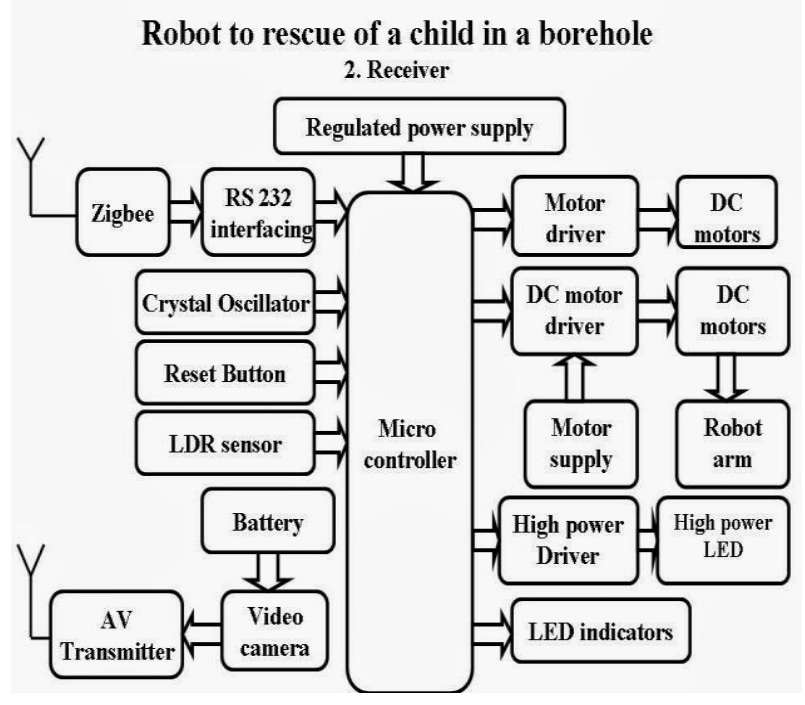

Figure 3.1 Block diagram 


\subsubsection{Robot Module}

This unit involves $12 \mathrm{~V}$ power supply, switch cushion and apparatus engines, security swell, solenoid valve. The switch cushion has four small scale changes associated with the microcontroller $\mathrm{I} / \mathrm{O}$ pins. One end of the switch is grounded and the other is associated with the microcontroller port. At the point when any switch is squeezed that specific port is grounded. The microcontroller is constantly observed these switches, progressively.

\subsubsection{Motor}

A DC engine is utilized to change over electrical vitality to mechanical vitality, normally through the collaboration of attractive field and current conveying channels. The turnaround process delivering electrical vitality from mechanical vitality is refined by an alternator, generator or dynamo. Many sorts of electric engines can be keeping running as generators, and the other way around. The contribution of DC engine is present/voltage and its yield is torque (speed). The DC engine has two essential parts the pivoting part that is known as the armature and the stationary part that incorporates curls of wire called the field loops. The stationary part is known as the stator.

\subsubsection{Solenoid Valve}

A solenoid valve is an electromechanical worked valve. The valve is controlled by an electric current through a solenoid: on account of a two-port valve the stream is turned on or off;

On account of a three-port valve, the outpouring is exchanged between the two outlet ports. In this venture solenoid valve is utilized to send oxygen to the safeguard tyke Solenoid valves are the most habitually utilized control components in fluidics. Their assignments are to stop, discharge, and measurements, circulate or, on the other hand blend liquids. They are found in numerous application ranges. Solenoids offer quick and safe exchanging, high dependability, long administration life, great medium similarity of the materials utilized, low control power and conservative plan.

\subsubsection{CCTV Camera}

Shut circuit TV (CCTV), otherwise called video reconnaissance, is the utilization of camcorders to transmit a flag to a particular place, on a constrained arrangement of screens. It contrasts from communicate TV in that the flag is not transparently transmitted; however it might utilize indicate point (P2P), indicate multipoint, or work remote connections. In mechanical plants, CCTV hardware might be utilized to watch parts of a procedure from a focal control room, for instance when nature is not appropriate for people. CCTV frameworks may work persistently or just as required to screen a specific occasion. CCTV camera associated with the TV tuner. It demonstrates the infant position inside the barrel and show on the TV.

\subsubsection{Advanced Digital Oxygen Supply System}

The breath of individuals will troublesome, if the rate of oxygen noticeable all around turns out to be under $18 \%$. So, we require a gadget to supply legitimate oxygen to the infant in the protect procedure. An oxygen concentrator is set on the surface of the drag well. It will naturally detect the deformity of oxygen at the save locale and it supplies the required oxygen. For this reason oxygen concentrator, an oxygen container of 200meters is set at first glance. The tube is sent alongside the robot to supply crisis oxygen to infant.

\subsubsection{Relay}

A transfer is an electrically worked switch. Many transfers utilize an electromagnet to mechanically work a switch, however other working standards are additionally utilized, for example, strong state transfers. Transfers are utilized where it is important to control a circuit by a low-control motion (with finish electrical disengagement amongst control and controlled circuits), or where a few circuits must be controlled by one flag. The main transfers were utilized as a part of long separation broadcast circuits as speaker. Transfers were utilized widely in phone trades and early PCs to perform intelligent operations. In this venture hand-off is utilized for controlling the engine, security swell, oxygen supply. In light of ON/OFF state it will work.

\subsubsection{Controller Unit}

The unit involves Microcontroller. This is a RISC (Reduced Instruction Set Computing) based Microcontroller having simple information channels, simple comparators and extra clock circuits. The Microcontroller stores the data received by the robot. The temperature detected by the robot is displayed on the LCD. Figure 3.3 shows block diagram of the AV receiver, the video caught by the CCTV camera is shown on a PC utilizing NI lab VIEW. The serial correspondence amongst microcontroller and $\mathrm{PC}$ is done through an interface.

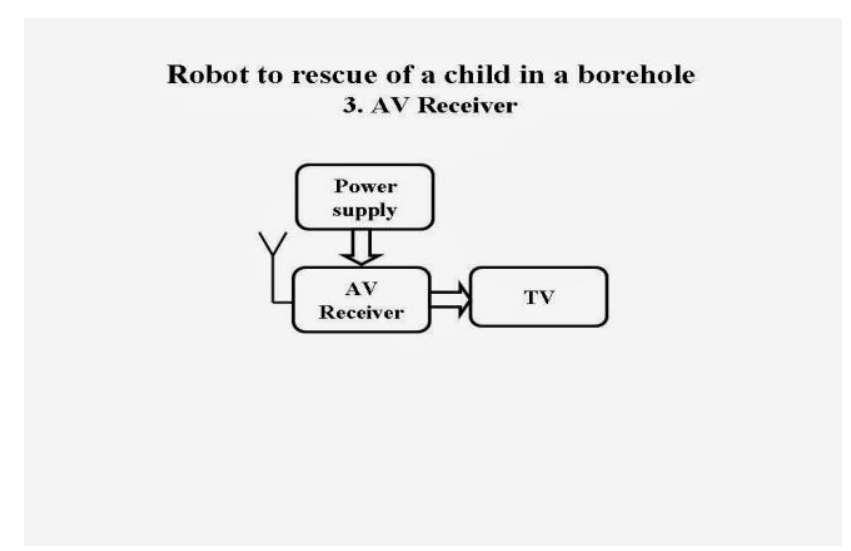

Figure 3.3 Block diagram of AV Receiver

\subsubsection{Display Unit}

For showing the data like temperature and smoke values, LCD $(16 \times 2)$ is utilized on each line this speck grid LCD show module holds 32 characters-16 on each line and has a green backdrop illumination with dark content.

\section{Experimental Results}

\subsection{Temperature Sensor}




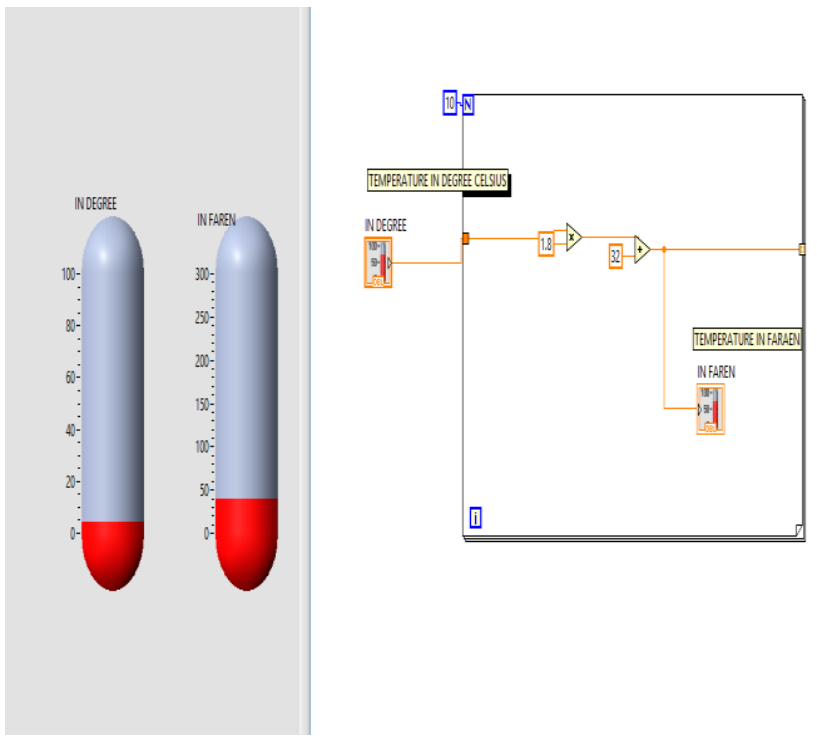

Figure 4.1 VI of Temperature Sensor

\subsection{Audio Recorder}

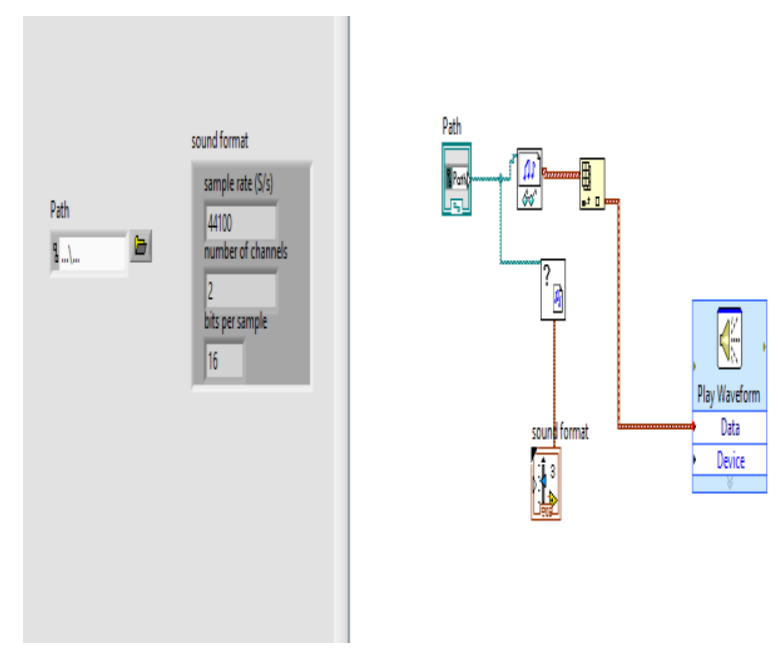

Figure 4.2 VI of Audio Recorder

\subsection{Altitude Sensor}
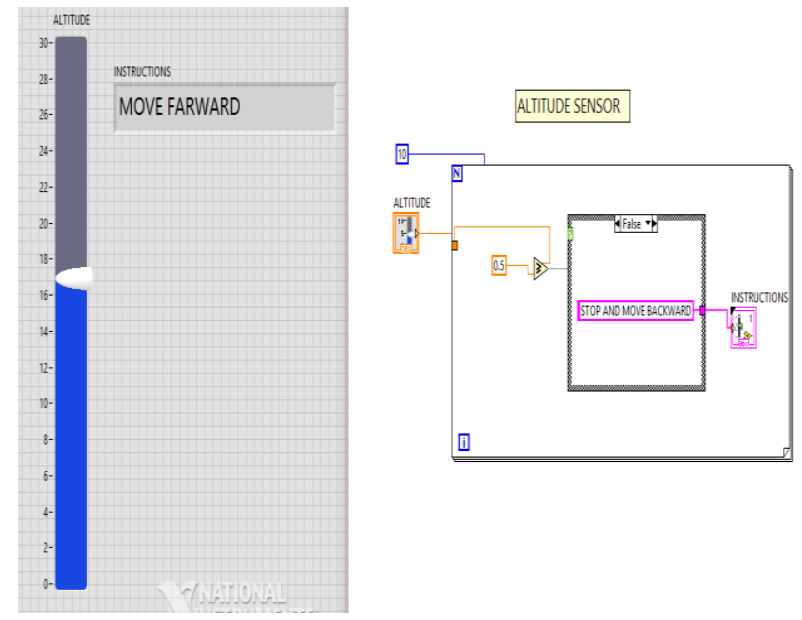

\subsection{Gas Sensor}
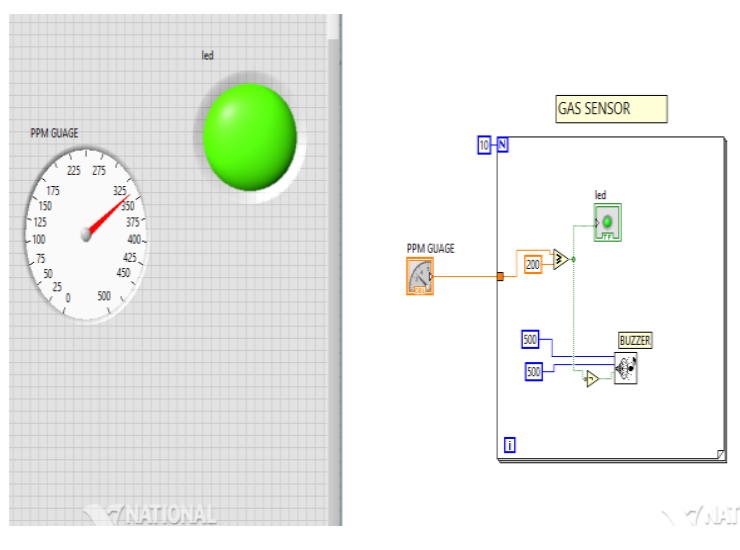

Figure 4.2 VI of Gas Sensor

\section{Conclusion}

The proposed system is mainly used for rescue child from bore well. The ZigBee transmitter, microcontroller, robot arm and the sensors perform their role in rescue operations. In past 10 years, a lot of lives have been lost due to falling in bore well because digging a pit beside the bore well is very tedious and time consuming process. The rescue operation team using this proposed technology will be safe and the rescue operation time will be reduced and will retain lives of many in future.

\section{References}

[1] Palwinder kaur, Ravinder kaur, Gurpeet singh.'Pipeline Inspection and Bore well Rescue Robot" IEEE, VOL 3,NO.4,April 2014.

[2] B.Bharathi, B.Suchitha Samuel "Design and Construction of Rescue Robot and Pipeline Inspection Using Zigbee”, International Journal of Scientific Engineering and Research (IJSER) Volume 1, Issue 1, September 2013.

[3] K.Saran, S.Vignesh, Marlon Jones Loius "Bore-well Rescue Robot" International Journal of Research Aeronautical and Mechanical Engineering (IJRAME) Vol 1, Issue 4, pg.61-80, April 2014.

[4] Manish Raj, P.Chakraborthy and G.C.Nandi "Rescue Robotics in Bore well Environment" Cornell University Library [VI] , 9 Jun 2014.

[5] V.Venmathi, E.Poorniya, S.Sumathi, "Borewell Rescue Robot" International Journal of Computer Applications, Volume 113,No.14, March 2015

[6] Sridhar Palaniswamy, "Life Saving Machine" The first international conference on Interdisplinary Research and Development,31 May-1 June 2011, Thailand.

[7] John Jose pottery, "Robot for bore well rescue", Amal Jothi College of Engineering Volume No. 10, June 2009. 